\title{
Antinociceptive local effect of the combination of dexketoprofen trometamol and chlorhexidine gluconate in a formalin test: an additive effect
}

\author{
Vicente Esparza-Villalpando, Daniel Chavarria-Bolaños ${ }^{2}$, Juan Ramón Zapata-Morales ${ }^{3}$, Antonio \\ Vértiz-Hernández ${ }^{4}$, Amaury Pozos-Guillén ${ }^{5 *}$
}

\begin{abstract}
${ }^{1}$ Materials Engineering and Science Doctorate Program, Universidad Autónoma de San Luis Potosi, México, ${ }^{2}$ Diagnostic and Surgical Sciences Department, Faculty of Dentistry, Universidad de Costa Rica, Costa Rica, ${ }^{3}$ Pharmacy Department, Universidad de Guanajuato, México, ${ }^{4}$ Coordinación Académica Región Altiplano, Universidad Autónoma de San Luis Potosi, México, ${ }^{5}$ Basic Sciences Laboratory, Faculty of Dentistry, Universidad Autónoma de San Luis Potosi, México
\end{abstract}

\begin{abstract}
A drug delivery system (DDS) with analgesic and antibacterial properties would be desirable for the local control of post-operatory pain and the prevention for surgical site infection (SSI). The objective of the present study was to evaluate the antinociceptive effect of the combination between dexketoprofen trometamol (DXT) and chlorhexidine gluconate (CHX) in the formalin pain model. Different doses of CHX were combined with DXT and were locally administered in rats paw simultaneously with 5\% formalin dilution. Flinches were documented and the antinociceptive effect was calculated. The area under the curve of each experimental group were calculated and the $\%$ of antinociception were compared. The groups of CHX and DXT showed similar antinociceptive effect. The combination groups (DXTCHX) showed higher antinociceptive effect that the one obtained with individual molecules. Besides the confirmation of DXT local antinociceptive properties, CHX also showed a positive effect; and an additive effect when combined with DXT.
\end{abstract}

Keywords: Antinociception/effects. Formalin test. Dexketoprofen trometamol. Chlorhexidine

\section{INTRODUCTION}

Pain is a complex response, caused by different elements that evokes the physiological, sensory and emotional pain process (Szallasi, 2010). Generally, is the consequence of local injury that affects the anatomical, biochemical and sensory equilibrium. When the damage is exposed to bacteria contamination, a dual etiology may trigger different pathways of the local inflammation (Arnstein, 2013).

Painful conditions can be controlled by the local application of analgesics, such as non-steroidal antiinflammatory drugs (NSAIDs), which inhibit cyclooxigenases (COXs) activity both central and peripherally (Derry et al., 2017). Dexketoprofen trometamol (DXT)

\footnotetext{
*Correspondence: A. Pozos-Guillén. Basic Sciences Laboratory, Facultad de Estomatología, Universidad Autónoma de San Luis Potosí. Av. Dr. Manuel Nava \#2, Zona Universitaria, CP 78290. San Luis Potosí, SLP, México. Phone number: 524448262357 X 5134. E-mail: apozos@uaslp.mx
}

is the water soluble $\mathrm{S}(+)$ enantiomer of the NSAID ketoprofen (Mauleón et al., 1996). Previous studies demonstrated the local effect of ketoprofen (Derry et al., 2015) and DXT (Isiordia-Espinoza et al., 2014; Sagir et al., 2013) and other studies showed analgesic efficacy of DXT in different post-operative conditions (EsparzaVillalpando et al., 2016; Rodríguez, Arbós, Amaro, 2008). The local administration of NSAIDs is desirable for elective interventions where the clinician can access directly to the lesion site. Theoretically, if the molecules are administered locally obtaining acceptable analgesia, then systemic intake can be reduced dramatically, thus decreasing the incidence of side-effects as well (Arnstein, 2013; Derry et al., 2017). In the surgical context, the procedure itself is susceptible to complications such as surgical site infection (SSI), especially in areas were microbial flora is abundant. This complication occurs in up to $30 \%$ of all surgical procedures (Bruce et al., 2001).

The use of skin antiseptics decrease the colonization of bacteria and therefore reduces the events of SSI (Lee 
et al., 2013). Chlorhexidine gluconate (CHX) is a topic antiseptic with antibacterial and antifungical clinical efficacy in different conditions, it is effective against gram-positive and gram-negative bacteria, some viruses and presents good tolerability and safety profile for the patient (Edmiston et al., 2010).

Animal pain models, can be used to test antinociceptive effect of drug combinations by evaluating the previous standardize response of rodents. The formalin test is a pain model used to study inflammatory pain by injecting noxious chemical irritant (formalin) into the paw of rodents (Szallasi, 2010; Krzyzanowska, Avendaño, 2012), allowing to determine not only the individual response of analgesic molecules; but the effect of certain combinations (showing either possible antagonism, synergism or additive effect of analgesic molecules under defined conditions). A drug delivery system (DDS) that allows analgesic and antibacterial properties would be desirable for the control of post-operatory pain, especially when the bacterial etiology is related to painful response (such as surgical procedures or post-surgical infections). Thus, the aim of the present study was to evaluate the antinociceptive effect of the combination of DXT and $\mathrm{CHX}$ in formalin pain model.

\section{MATERIAL AND METHODS}

\section{Animals}

Thirty six female Wistar rats (6 per group) of 6-7 weeks of age (200-250 g), were used in the experiment. The animals were obtained from the Animal Center of the Guanajuato University and were housed on a $12 \mathrm{~h}$ light-dark cycle at $24 \pm 2{ }^{\circ} \mathrm{C}$, with free access to food and water. On the day of the experiment, animals were acclimatized to the laboratory conditions for at least 2 hours before the test. All animals were used once and were sacrificed immediately after the test. The study was carried out according to the Official Mexican Norm NOM-062-ZOO-1999 (Technical specifications for the production, care, and use of laboratory animals) and the Guidelines on Ethical Standards for Investigation of Experimental Pain in Animals (Zimmermann, 1983). Animals were used once during the protocol and then sacrificed in $\mathrm{CO}_{2}$ chamber at the end of each experiment. DXT (Stein Labs ${ }^{\circledR}$, San José, Costa Rica) was dissolved at the moment of the experiment in des-ionized water, obtaining a concentration of $12 \mathrm{mg} / \mathrm{mL}$. CHX (Sigma Aldrich ${ }^{\circledR}$, EUA), 20\% water solution, was dissolved in des-ionized water in 3 different concentrations $(0.02 \%$, $0.3 \%$ and $2.5 \%$ respectively).

\section{Measurement of nociceptive response}

Antinociception was assessed by the formalin test. The rats were placed in transparent plastic cylindrical chambers with mirrors placed in a $45^{\circ}$ angle to assure complete viewing of the injected paw. The rats were injected into the dorsal surface of the hind paw with $50 \mu \mathrm{L}$ of dilute formalin (5\%) alone or mixed with the appropriated concentrations of formulations using a 30 -gauge needle. The rats were observed for nociceptive behavior immediately after formalin injection. All measurements were made by an independent blinded evaluator. Nociceptive behavior was quantified as the number of flinches of the injected paws in one minute (min) periods, every $5 \mathrm{~min}$ up to $60 \mathrm{~min}$ after injection. Time-courses of nociceptive response for each group were constructed as mean number of flinches in each time. For the two phases of formalin test, the areas under the curve (AUC) were calculated by trapezoidal method (Allison et al., 1995): the first phase included the measures of the minutes 0-15; and the second phase the minutes 15-60. Group-response curves for each phase were constructed using six animals for each group. The percentage of antinociception (\%AN) was calculated as follow:

$$
\% A N=\frac{A U C_{\text {Formalin }}-A U C_{\text {Treatment group }}}{A U C_{\text {Formalin }}} \times 100
$$

\section{Experimental design}

The animals were injected with a total solution of $50 \mu \mathrm{L}$ per experiment, containing fixed volumes that kept the following concentrations: formalin 5\%, DXT $12 \mathrm{mg} / \mathrm{mL}$ and CHX (with different concentrations as mentioned before). Proportions v/v are shown in the Table I. All solutions were prepared freshly the day of the test and were put together directly in the same syringe right before its application.

\section{Statistical analysis}

The antinociceptive responses are presented as Mean \pm Standard Error (S.E.M.), the AUC $/ \%$ AN difference between experimental groups was assessed by ANOVA with planned contrast: a) G1 vs G2, G3, G4, G5, G6; contrast b) G2 vs G3, G4, G5, G6; contrast c) G3 vs G4, G5, G6; contrast d) G4 vs G5, G6 and contrast e) G5 vs G6 and the assumption of normality was assessed by Shapiro-Wilks test. The same contrasts were performed to both phase 1 and phase 2. All analysis were performed by statistical software $\mathrm{R}$ ver. 3.4.0. 
TABLE I - Description of the experimental groups

\begin{tabular}{lcccc}
\hline Groups & $\boldsymbol{\mu L}$ of Formalin & $\boldsymbol{\mu L}$ of DXT $\dagger$ & $\boldsymbol{\mu L}$ of CHX $\dagger$ & \% of CHX \\
\hline G1 (Control) & 50 & - & - & - \\
G2 (DXT only) & 25 & 25 & - & - \\
G3 (CHX 0.3\% only) & 25 & - & 25 & 0.3 \\
G4 (DXT + CHX 0.02\%) & 25 & 12.5 & 12.5 & 0.02 \\
G5 (DXT + CHX 0.3\%) & 25 & 12.5 & 12.5 & 0.3 \\
G6 (DXT + CHX 2.5\%) & 25 & 12.5 & 12.5 & 2.5 \\
\hline
\end{tabular}

$\dagger$ Dexketoprofen trometamol, $\uparrow$ Chlorhexidine

\section{RESULTS}

The test for normality (Shapiro-Wilks) showed data distribution not different to normal $(\mathrm{P}>0.05)$. The time course of the nociceptive response is shown on Figure 1. The formalin test showed two well defined phases, the phase 1 (1-11 minutes) and the phase 2 (21-61 minutes) representing tonic acute pain and inflammatory pain respectively.

The \%AN and the p-values of different comparisons are shown on the Figures 2 and 3. Control group showed the lower value of \% AN in both phases, the group DXT only (G2), and the other groups not showed statistical difference. Additionally, the group of CHX only (G3), show similar antinociceptive effect to DXT only (G2). The group of the combination between DXT $+\mathrm{CHX} 0.02 \%(\mathrm{G} 4)$ showed lower antinociceptive effect than groups G5 and
G6, but only on the phase 1. The group of CHX only (G3) showed lower antinociceptive effect than the combination groups (G4, G5 and G6), but only on the phase 2 .

\section{DISCUSSION}

This work confirmed the antinociceptive effect of DXT when is locally administered in the formalin test, using the $50 \%$ effective dose of DXT previously reported by Isiordia et al. (2014). The local use of NSAID'S can provide acceptable pain relief, commonly reported in acute conditions. Ketoprofen is one of the molecules with this effect (Derry et al., 2015), supporting the logical election of DXT for this investigation. Moreover, this work focuses on the antinociceptive effect of the combination between DXT and CHX in the formalin model of inflammatory pain. The concentrations used in this study were selected

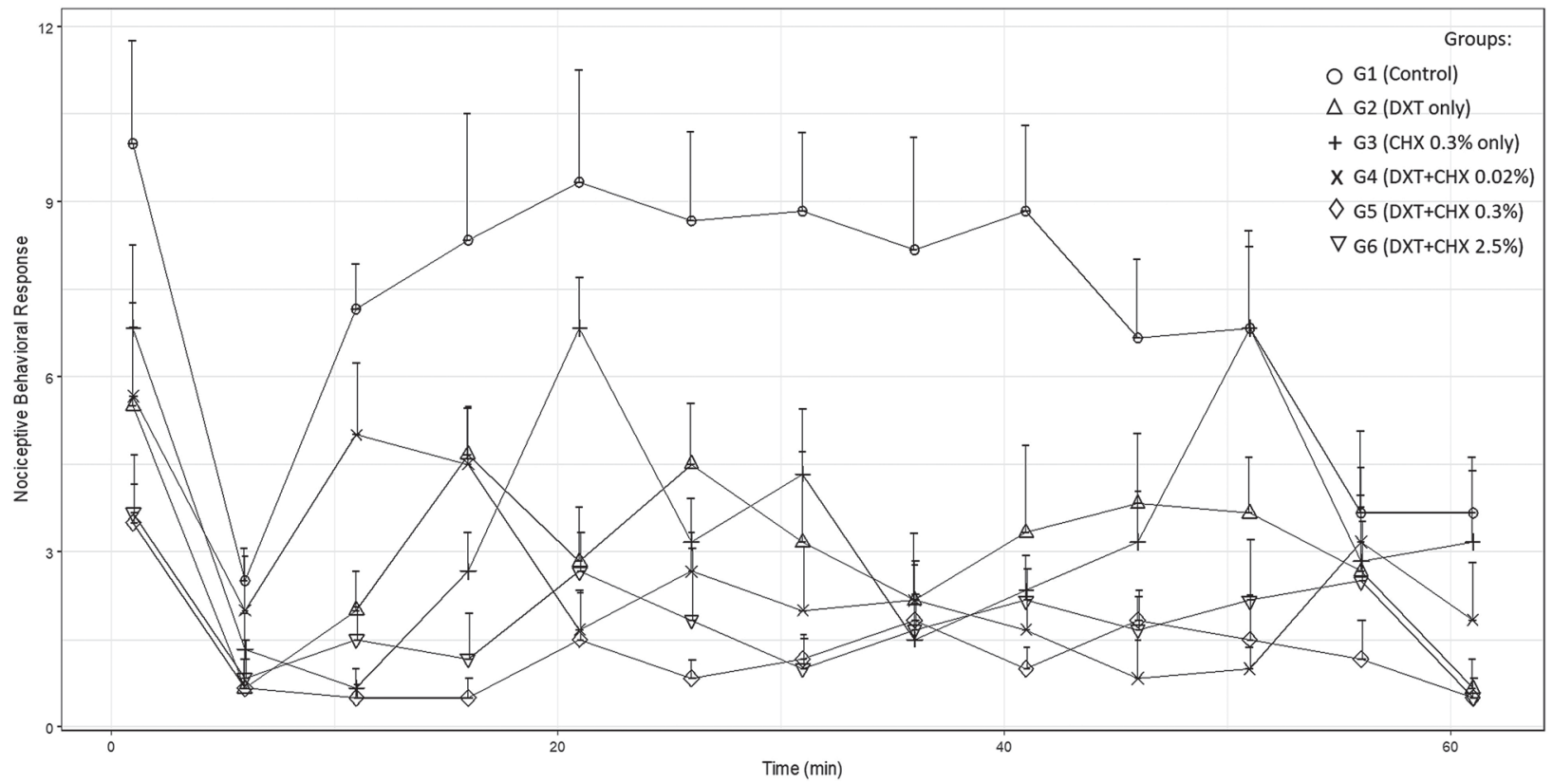

FIGURE 1 - Time course of the nociceptive behavioral response of the experimental groups ( $\mathrm{n}=6$ rats each group). Each symbol represents the mean with S.E.M., over the 60 min post-injection observation period. 


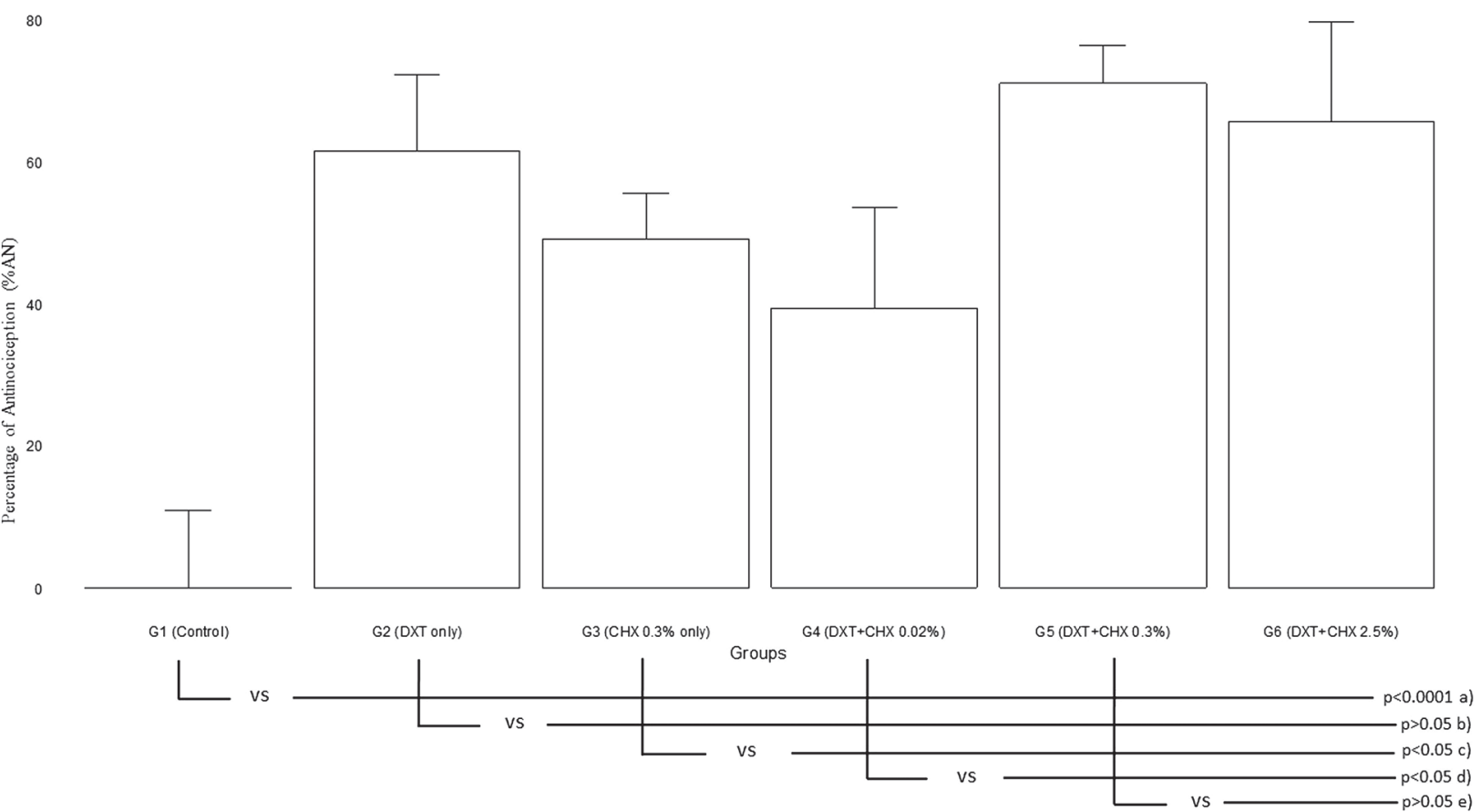

FIGURE 2 - Antinociceptive effects (\%AN) of control and experimental groups in the first phase of formalin test. Data are present in Mean \pm S.E.M. of $n=6$ rats for each group. The letters represent the planned contrast ANOVA: a) G1 vs G2, G3, G4, G5, G6; b) G2 vs G3, G4, G5, G6; c) G3 vs G4, G5, G6; d) G4 vs G5, G6 and e) G5 vs G6.
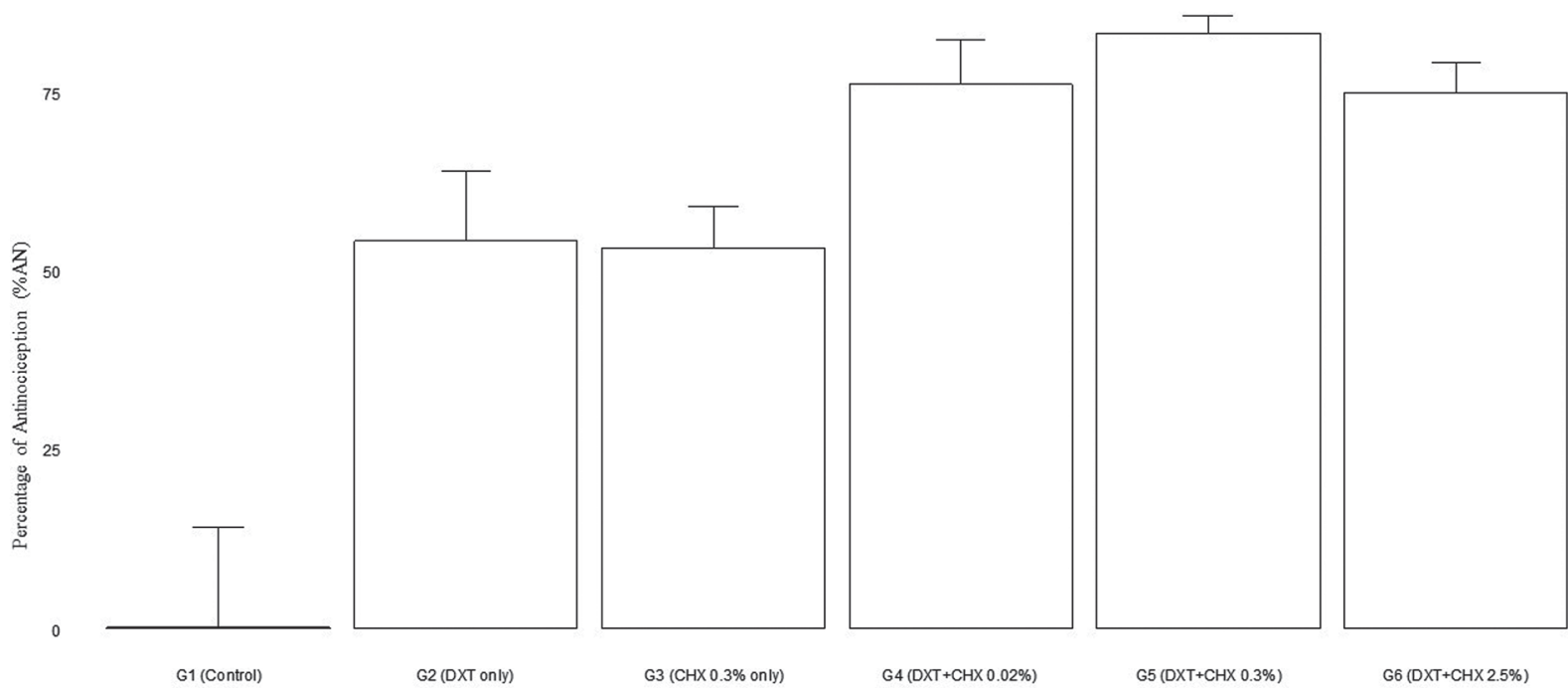

G1 (Control)

vs
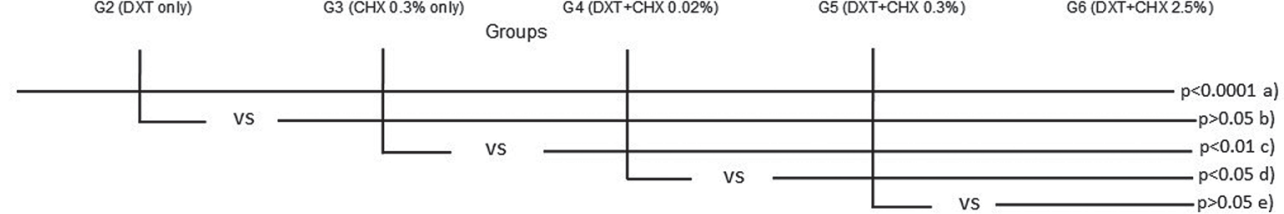

FIGURE 3 - Antinociceptive effects (\%AN) of control and experimental groups in the second phase of formalin test. Data are present in Mean \pm S.E.M. of $\mathrm{n}=6$ rats for each group. The letters represent the planned contrast ANOVA: a) G1 vs G2, G3, G4, G5, G6; b) G2 vs G3, G4, G5, G6; c) G3 vs G4, G5, G6; d) G4 vs G5, G6 and e) G5 vs G6.

based on previously reported minimal inhibitory concentration (MIC) for CHX (Edmiston et al., 2010; Hope, Wilson, 2004), since they are considered noncytotoxic (Faria et al., 2007; Faria et al., 2009; Lee et al.,
2010; Li et al., 2009). As mentioned before, beyond the classic employments of formalin test, this research tool also can discard possible antagonistic reactions between the components of new pharmacological combinations; in 
our case, it was selected as the departure point to propose an analgesic/antiseptic DDS.

The behavioral response showed the classical two phases induced by the formalin test (Gonzalez et al., 2011; Miranda et al., 2011; Krzyzanowska, Avendaño, 2012). Phase 1 results from a direct stimulation of nociceptors and phase 2 involves a period of sensitization during the inflammatory process through peripheral mechanisms (Le Bars, Gozariu, Cadden, 2001). The expected result was that the combination between both did not showed a decreased antinociceptive effect, conversely, the combination showed an increased antinociceptive effect than the drug alone, but these differences were not significative.

To our knowledge, an antinociceptive effect related to local application of CHX has not yet been reported. Haraji and Rakhshan showed in a clinical trial an analgesic effect of the CHX gel in post-operative pain condition. As the authors hypothesized, the antiseptic feature of the CHX is possibly related to the reduction in painful inflammation mediators caused by bacteria (Haraji, Rakhshan, 2015). However, these results were inconclusive and more studies are needed to confirm these findings. Even when such conclusion may be indirectly related to the present results, they cannot be correlated since the nociceptive response of the formalin test is related to a noxious chemical stimulus and no to bacterial presence. In this context, the possible mechanism involved in the antinociceptive or analgesic response of the CHX should be analyze in deeper specialized models, including the analysis of specific pain pathways, expression of potential mediators or even the agonism/antagonism of certain receptors. Due to its chemical composition and ionic charge, $\mathrm{CHX}$ molecules may modulate the nervous conduction responsible of pain transmission, or even may contribute by blocking specific peripheral ionic channels. The bactericidal effect of CHX is a result of the binding of the $\mathrm{CHX}$ cationic molecules to negatively charged bacterial cell walls and extramicrobial complexes (McDonnell, Russell, 1999). A possible reason of the antinociceptive effect observed may involve the positively charged CHX interacting with the membranes of the peripheral nerve endings, thus creating the modulation of the action potential evoked by the formalin. However, any of these hypotheses must be meticulously analyzed in new experiments.

The surgical procedures involve wounds that will heal by primary or secondary intention. In the procedures involving "clean" cavities, the surgical infection prevalence is around $3 \%$ to $5 \%$; but, when the procedures involve infected sites, dirty or necrotic tissue; the surgical infections increase up to $10 \%$ to $30 \%$ (White, 2009). The oral cavity is considered clean/contaminated and the risk for surgical infection is latent. The wound infection results from the dynamic interactions between the presence of pathogens and the susceptibility of the host. The pathways of the surgical infection are complex; however, the most common signs are erythema, pain, local temperature and swelling (Gardner, Frantz, Doebbeling, 2001).

The presence of pain is part of the local inflammatory reaction, and thus, it will be expected to decrease at the same rhythm that the etiology is controlled and the inflammation is auto-limited. To increase the quality of life of the patients, analgesic/antiseptic DDS will play a dual role, by modulating the normal painful response of inflammation while favoring the local septic ethology. When pain is poorly controlled, a pain-related infection would reduce the immune response to infection. Therefore, the control of pain is as important as the treatment of infection itself (White, 2009). The use of CHX could provide an extra analgesic effect in combination with DXT and at the same time covering the antimicrobial role to prevent SSI.

Since our results not only demonstrated that the presence of CHX didn't affect the local antinociceptive effect of DXT (confirmed in our results), but the molecule itself showed to have antinociceptive capacity, and the presence of an additive effect between both molecules; a new favorable result must be concluded. Further efforts will be addressed not only to fully understand this hypothesis and the mechanisms involved, but to include new experiments analyzing decreasing combinatory doses in order to perform an isobolographic analysis (Tallarida, 2001), looking for possible pharmacological synergism.

\section{CONFLICT OF INTEREST}

All authors declare no competing interests.

\section{ACKNOWLEDGMENTS}

Vicente Esparza-Villalpando is a CONACYT fellow $599447 / 326741$. This study was supported partially by UASLP C18-FAI-05-50.50 grant.

\section{REFERENCES}

Allison DB, Paultre F, Maggio C, Mezzitis N, Pi-Sunyer XF. The use of areas under curves in diabetes research. Diabetes Care. 1995;18(2):245-50.

Arnstein PM. The future of topical analgesics. Postgrad Med. 2013;125(4):34-41. 
Bruce J, Russell EM, Mollison J, Krukowski ZH. The measurement and monitoring of surgical adverse events. Health Technol Assess (Rockv). 2001;5(22):1-194.

Derry S, Moore RA, Gaskell H, Mcintyre M, Wiffen PJ. Topical NSAIDs for acute musculoskeletal pain in adults. Cochrane Database Syst Rev. 2015;6:CD007402.

Derry S, Wiffen PJ, Kalso EA, Bell RF, Aldington D, Phillips T, et al. Topical analgesics for acute and chronic pain in adults - an overview of Cochrane Reviews. In S. Derry (editor). Cochrane Database Syst Rev. 2017;5:CD008609.

Edmiston CE, Okoli O, Graham MB, Sinski S, Seabrook GR. Evidence for using chlorhexidine gluconate preoperative cleansing to reduce the risk of surgical site infection. AORN J. 2010;92(5):509-18.

Esparza-Villalpando V, Chavarria-Bolaños D, Gordillo-Moscoso A, Masuoka-Ito D, Martinez-Rider R, Isiordia-Espinoza M, et al. Comparison of the analgesic efficacy of preoperative/ postoperative oral dexketoprofen trometamol in third molar surgery: A randomized clinical trial. J Craniomaxillofac Surg. 2016;44(9):1350-5.

Faria G, Celes MRN, De Rossi A, Silva LAB, Silva JS, Rossi MA. Evaluation of chlorhexidine toxicity injected in the paw of mice and added to cultured 1929 fibroblasts. J Endod. 2007;33(6):715-22.

Faria G, Cardoso CRB, Larson RE, Silva JS, Rossi MA. Chlorhexidine-induced apoptosis or necrosis in L929 fibroblasts: A role for endoplasmic reticulum stress. Toxicol Appl Pharmacol. 2009;234(2):256-65.

Gardner SE, Frantz RA, Doebbeling BN. The validity of the clinical signs and symptoms used to identify localized chronic wound infection. Wound Repair Regen. 2001;9(3):178-86.

Gonzalez C, Zegpi C, Noriega V, Prieto JC, Miranda HF. Synergism between dexketoprofen and meloxicam in an orofacial formalin test was not modified by opioid antagonists. Pharmacol Rep. 2011;63(2):433-40.

Haraji A, Rakhshan V. Chlorhexidine gel and less difficult surgeries might reduce post-operative pain, controlling for dry socket, infection and analgesic consumption: A splitmouth controlled randomised clinical trial. J Oral Rehabil. 2015;42(3):209-19.
Hope CK, Wilson M. Analysis of the effects of chlorhexidine on oral biofilm vitality and structure based on viability profiling and an indicator of membrane integrity. Antimicrob Agents Chemother. 2004;48(5):1461-8.

Isiordia-Espinoza MA, Pozos-Guillén A, Pérez-Urizar J, Chavarría-Bolaños D. Involvement of nitric oxide and ATPsensitive potassium channels in the peripheral antinoceptive action of a tramadol - dexketoprofen combination in the formalin test. Drug Dev Res. 2014;75(7):449-54.

Krzyzanowska A, Avendaño C. Behavioral testing in rodent models of orofacial neuropathic and inflammatory pain. Brain Behav. 2012;2(5):678-97.

Le Bars D, Gozariu M, Cadden SW. Animal models of nociception. Pharmacol Rev. 2001;53(4):597-652.

Lee TH, Hu CC, Lee SS, Chou MY, Chang YC. Cytotoxicity of chlorhexidine on human osteoblastic cells is related to intracellular glutathione levels. Int Endod J. 2010;43(5):430-5.

Lee I, Agarwal RK, Lee BY, Fishman NO, Umscheid CA Systematic review and cost analysis comparing use of chlorhexidine with use of iodine for preoperative skin antisepsis to prevent surgical site infection. Infect Control Hosp Epidemiol. 2013;31(12):2-21.

Li YC, Kuan YH, Lee SS, Huang FM, Chang YC. Cytotoxicity and genotoxicity of chlorhexidine on macrophages in vitro. Environ Toxicol. 2009;24(3):296-303.

Mauleón D, Artigas R, García ML, Carganico G. Preclinical and clinical development of dexketoprofen. Drugs. 1996;52(5):2446.

McDonnell G, Russell AD. Antiseptics and disinfectants: activity, action, and resistance. Clin Microbiol Rev. 1999;12(1):147-79.

Miranda HF, Noriega V, Sierralta F, Prieto JC. Interaction between dexibuprofen and dexketoprofen in the orofacial formalin test in mice. Pharmacol Biochem Behav. 2011;97(3):423-7.

Rodríguez MJ, Arbós RM, Amaro SR. Dexketoprofen trometamol: clinical evidence supporting its role as a painkiller. Expert Rev Neurother. 2008;8(11):1625-40.

Sagir O, Sunay FB, Yildirim H, Aksoz E, Ozaslan S, Koroglu A, et al. Evaluation of the effects of dexketoprofen trometamol on knee joint: an in vivo \& in vitro study. Indian J Med Res. 2013;138(6):912-8. 
Szallasi, A. Analgesia: Methods and Protocols. Analgesia: methods and protocols. New York: Springer; 2010. p.557.

Tallarida RJ. Drug synergism: its detection and applications. J Pharmacol Exp Ther. 2001:298(3):865-72.

White RJ. Wound infection-associated pain. J Wound Care. 2009;18(6):245-49.
Zimmermann, M. Ethical guidelines for investigations of experimental pain in conscious animals. Pain. 1983;16(2):10910.

Received for publication on $05^{\text {th }}$ December 2017 Accepted for publication on $24^{\text {th }}$ December 2017 